\title{
Desempenho de Frangos de Corte nas Fases de Crescimento e Final Alimentados com Rações Contendo Soja Integral Extrusada em Diferentes Temperaturas ${ }^{1}$
}

\author{
Francisco Nairson de Oliveira ${ }^{2}$, Fernando Guilherme Perazzo Costa ${ }^{3}$, José Humberto Vilar da \\ Silva $^{4}$, Patrícia Araújo Brandão ${ }^{5}$, Valene da Silva Amarante Júnior ${ }^{5}$, Germano Augusto Jerônimo \\ do Nascimento ${ }^{6}$, Leilane Rocha Barros ${ }^{6}$
}

\begin{abstract}
RESUMO - O efeito da temperatura de extrusão da soja integral (SIE) sobre o desempenho e as características de carcaça de frangos de corte em diferentes fases de criação foi avaliado neste experimento. As rações, isonutritivas, foram formuladas para atender às exigências nutricionais das aves nas fases de crescimento (22 a 35 dias) e final ( 36 a 45 dias de idade). Os tratamentos experimentais consistiram de quatro temperaturas de extrusão da soja integral $\left(125,130,135\right.$ e $\left.140^{\circ} \mathrm{C}\right)$, com nove repetições de 30 aves. As variáveis avaliadas foram consumo de ração (CR), ganho de peso (GP), conversão alimentar (CA) e características da carcaça (rendimentos de perna e de peito e gordura abdominal). Em ambas as fases, os tratamentos não apresentaram efeito significativo sobre as variáveis estudadas. Os resultados indicam que o processamento da soja integral com extrusão à temperatura de 125 a $140^{\circ} \mathrm{C}$ pode ser utilizado visando à utilização do extrusado em dietas para frangos de corte.
\end{abstract}

Palavras-chave: carcaça, desempenho, frangos de corte, soja integral extrusada, tratamento térmico

\section{Effects of Full-Fat Extruded Soybean at Different Temperatures on Performance of Broiler Chicks in the Growing and Final Phases}

\begin{abstract}
The effects of full-fat extruded soybean at different temperatures (FES) on performance of broiler chickens were evaluated in this trial. Isonutritive diets were formulated to meet broiler requirements in the growing (22-35 days old) and finishing ( 36 - 45 days old) phases. The experimental treatments consisted of four soybean extrusion temperatures $\left(125,130,135\right.$ and $\left.140^{\circ} \mathrm{C}\right)$ with nine replicates of 30 birds. The variables investigated were: feed intake, weight gain, feed:gain ratio and carcass composition (leg yield, breast meat yield and abdominal fat). In both phases, no significant effect of the treatments on the variables was detected. Soybean extruded at temperature of 125 to $140^{\circ} \mathrm{C}$ can be used in broiler chickens diets after 22 days old.
\end{abstract}

Key Words: carcass, performance, broiler chicks, full-fat extruded soybean, thermic treatment

\section{Introdução}

O progresso na nutrição de aves nos últimos 50 anos é atribuído a vários fatores, como o uso de vitaminas sintéticas e enzimas e a utilização de aminoácidos (incluindo proteína ideal) em vez de proteína bruta. Vale ressaltar o desenvolvimento de programas alimentares para satisfazer às exigências específicas das aves durante o ciclo de produção e de linhagens genéticas, além da adição de macro e micro elementos às dietas, da relação energia:proteína, do desenvolvimento genético de grãos e do processamento de alimentos.

A soja (Glycine max) representa mais da metade do total de grãos de leguminosas produzidos no mundo e, nos últimos 30 anos, tornou-se a principal fonte de proteína vegetal. Na sua industrialização, o farelo é gerado com um rendimento de $76,5 \%$, representando dois terços dos farelos protéicos consumidos na alimentação animal (Rostagno et al., 2004). Os atributos do farelo que o tornam o ingrediente preferencial na alimentação de monogástricos são a alta concentração de proteína, o perfil e o nível de aminoácidos, especialmente a lisina, e o alto valor energético.

Contudo, a utilização da soja na alimentação de não-ruminantes requer cuidados quanto ao adequado tratamento térmico, que inibe os fatores antinutricionais contidos nesta leguminosa. Os dois principais parâmetros de qualidade da soja referem-se ao nível de atividade de urease, mantido na faixa de 0,05 a 0,2

\footnotetext{
${ }^{1}$ Parte da Dissertação de Mestrado em Zootecnia do primeiro autor-PPGZ/UFPB/CCA/Areia-PB.

2 Prof. da Escola Agrot. Fed. de Souza-PB e aluno do PPGZ/UFPB/CCA/Areia-PB.

${ }^{3}$ Prof. do Departamento de Zootecnia e Pós-Graduação-DZ/PPGZ/UFPB/CCA/Areia-PB (fperazzo@cca.ufpb.br).

${ }^{4}$ Prof. do CFT-Bananeiras-PB/UFPB e Pós-Graduação-DZ/PPGZ/UFPB/CCA/Areia-PB.

${ }^{5}$ Aluno do Doutorado do Programa de Doutorado Integrado em Zootecnia-PDIZ/UFPB/CCA/Areia-PB.

${ }^{6}$ Alunos de Mestrado do Programa de Pós-Graduação em Zootecnia-PPGZ/UFPB/CCA/Areia-PB.
} 
unidades de $\mathrm{pH}$, e ao índice de solubilidade protéica de 80 a $85 \%$, que depende das temperaturas utilizadas nas etapas industriais.

Os principais fatores antinutricionais são os que reduzem a ação da tripsina e da quimiotripsina, causando perda no desempenho desses animais (Bond \& Smith, 1988). Segundo Kakade et al. (1973), citados por Han et al. (1991), 40\% da perda do desempenho em aves consumindo soja crua pode ser creditado ao efeito do inibidor Kunitz.

Os inibidores de tripsina são compostos protéicos que se complexam à tripsina (enzima pancreática), prejudicando a digestão das proteínas alimentares já desdobradas pela ação da pepsina, e, conseqüentemente, a quebra das cadeias protéicas que liberariam os aminoácidos para absorção intestinal (Butolo, 2001). Os níveis desses inibidores em grãos de soja crua variam de 30 a $50 \mathrm{mg} / \mathrm{kg}$. O tratamento térmico é considerado adequado quando promove a destruição de 80 a 90\% desta atividade (Moreira, 2000).

Ao contrário do que se pensava, a soja crua possui também quantidade apreciável de polissacarídeos nãoamiláceos (PNA's) na forma de pectinas, hemiceluloses e dos oligassacarídeos rafinose e estaquinose. Além desses PNA's, fatores antinutricionais como inibidores de proteases e lectinas estão amplamente distribuídos na soja e não podem ser degradados pelo sistema digestivo das aves (Jeroch \& Dâniche, 1995).

Para melhorar a aceitabilidade da soja, é necessário tratamento térmico adequado para inativação dos componentes antinutricionais, melhorando a aceitabilidade da soja, em razão da formação de substâncias resultantes da condensação de glicídios e aminoácidos (Liener, 1986).

Ludke et al. (2004) relataram que a soja integral processada, por apresentar as vantagens do farelo, associadas ao elevado valor energético decorrente da presença do óleo no grão, envolve um potencial econômico a ser explorado na produção de monogástricos. Entre as principais alternativas para o processamento, encontram-se a extrusão, expansão, autoclavagem, micronização e tostagem. Segundo Said (1999), os processos industriais que utilizam temperaturas de 110 a $120^{\circ} \mathrm{C}$ são considerados ideais em termos de manutenção da qualidade protéica da soja.

Este trabalho foi realizado com o objetivo de se analisar o efeito da temperatura de extrusão da soja integral sobre o desempenho e as características de carcaça de frangos de corte nas fases de crescimento e final.

\section{Material e Métodos}

O experimento foi conduzido no Setor de Avicultura da Universidade Federal da Paraíba, Campus II, localizado em Areia/PB, no período de 27 de abril a 20 de maio de 2002. Foram utilizados 1.080 frangos de corte machos da linhagem Ross, vacinados contra a doença de Marek e, posteriormente, contra a de Newcastle e Gumboro. As aves foram alojadas em 36 boxes $\left(2,70 \mathrm{~m}^{2}\right)$ de alvenaria com piso de cimento, coberto com cama de bagaço de cana-de-açúcar triturado e desidratado. Foram utilizados comedouros tubulares e bebedouros pendulares, onde foram fornecidas ração e água à vontade. As aves foram submetidas a um programa de luz contínuo (24 horas de luz natural + artificial).

As rações, formuladas para atender às exigências nutricionais das aves, de acordo com cada fase de criação (Rostagno et al., 2000), foram fornecidas na forma farelada (Tabela 1). O ingrediente soja integral extrusada recebeu quatro temperaturas diferentes $\left(125^{\circ} \mathrm{C}-\mathrm{T}_{1}, 130^{\circ} \mathrm{C}-\mathrm{T}_{2}, 135^{\circ} \mathrm{C}-\mathrm{T}_{3}\right.$ e $\left.140^{\circ} \mathrm{C}-\mathrm{T}_{4}\right)$ em uma extrusora Calibras modelo Extrusoy $1500 \mathrm{com}$ cinco anéis no canhão. A temperatura de $135^{\circ} \mathrm{C}\left(\mathrm{T}_{3}\right)$ corresponde ao tratamento utilizado comercialmente. Os tratamentos consistiram de níveis fixos de inclusão da soja integral extrusada na ração.

No final de cada fase de criação, foram avaliados o consumo de ração, o ganho de peso e a conversão alimentar. Ao encerrar a fase final, foram separadas quatro aves por parcela, que foram submetidas a jejum de 8 horas e abatidas, para avaliação dos rendimentos de carcaça eviscerada (sem pena e vísceras comestíveis e não-comestíveis), de peito e de perna (coxa e sobrecoxa) e da gordura abdominal.

O delineamento experimental utilizado foi o inteiramente casualizado, constituído por quatro tratamentos da soja integral extrusada $\left(125,130,135\right.$ e $\left.140^{\circ} \mathrm{C}\right)$ e nove repetições com 30 aves por unidade experimental, totalizando 1.080 aves. As análises de variância do experimento foram realizadas de acordo com o seguinte modelo matemático:

$$
\mathrm{Y}_{\mathrm{ik}}=\mu+\mathrm{T}_{\mathrm{i}}+\mathrm{e}_{\mathrm{ik}}
$$

em que $\mathrm{Y}_{\mathrm{ik}}=$ observação relativa a i-ésima temperatura de extrusão de soja integral na k-ésima parcela experimental; $\mu=$ média geral do experimento; $T_{i}=$ efeito da i-ésima temperatura de extrusão da soja integral, sendo $\mathrm{i}=125,130,135$ e $140^{\circ} \mathrm{C} ; \mathrm{e}_{\mathrm{ik}}=$ erro aleatório associado a cada observação. 
Tabela 1 - Composições percentual e calculada das rações experimentais

Table 1 - Ingredient calculated composition (\%) of experimental diets

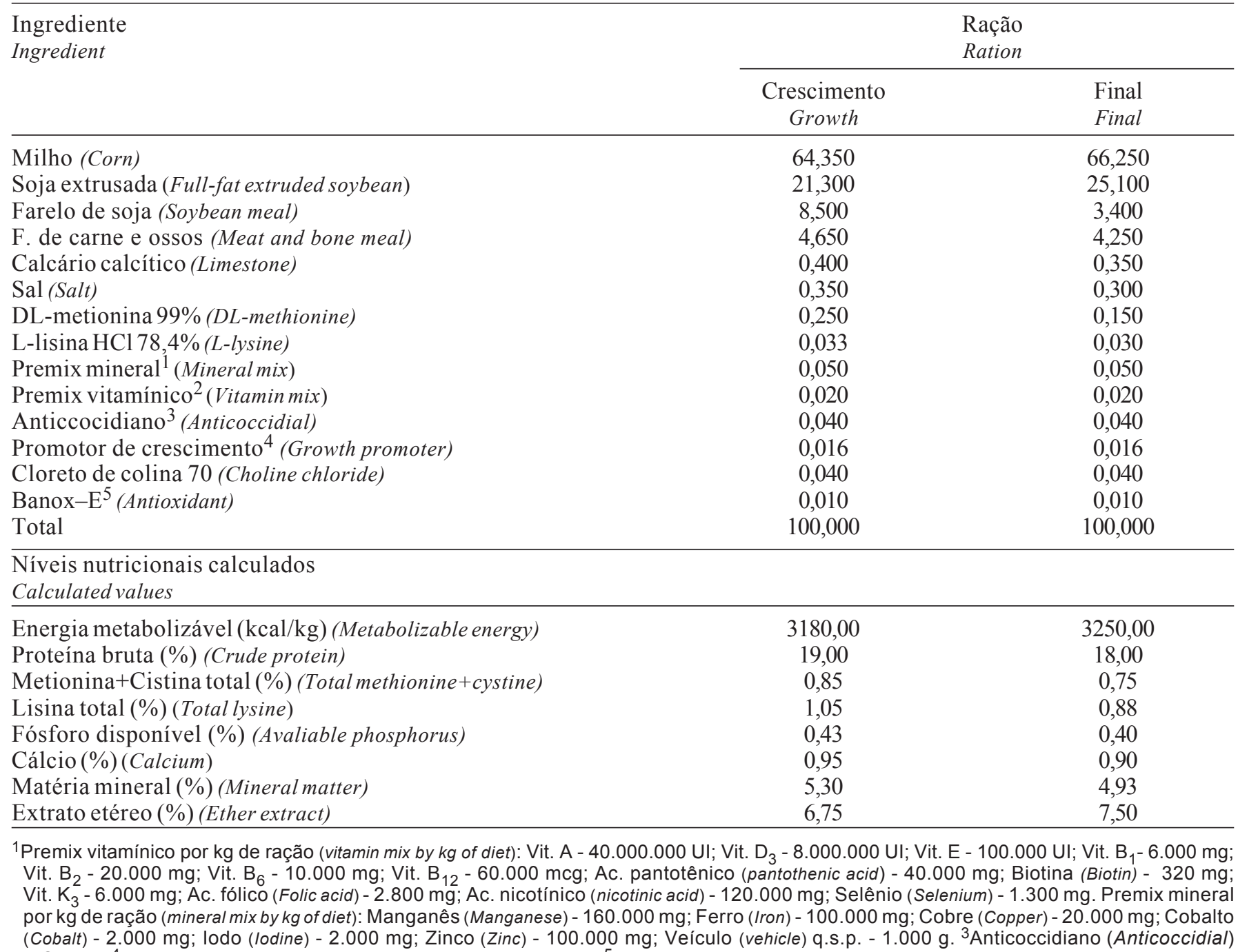

- Coban. ${ }^{4}$ Promotor de crescimento (Growth promoter) - Enradin. ${ }^{5}$ Antioxidante (Sinox) - Banox-E.

As análises estatísticas das características foram avaliadas por intermédio do Programa SAEG 8.0 (1999). As estimativas dos graus de liberdade dos tratamentos (temperaturas) foram decompostos nos efeitos lineares e quadráticos. Na escolha do modelo, considerou-se o nível de significância $(\mathrm{P}<0,05)$, o coeficiente de determinação $\left(\mathrm{r}^{2}\right)$ e a resposta biológica das aves aos tratamentos experimentais.

\section{Resultados e Discussão}

A mortalidade dos frangos de corte na fase de crescimento (22 a 35 dias de idade) foi de $0,28 \%$. Nessa fase, não foi observado efeito significativo $(\mathrm{P}>0,05)$ do aumento de temperatura de extrusão da soja integral sobre o consumo de ração (CR), o ganho de peso (GP) e a conversão alimentar (CA) (Tabela 2).
Os resultados indicam que, provavelmente em razão do amadurecimento do sistema enzimático, as aves mais velhas são menos sensíveis às baixas temperaturas de extrusão da soja. Marsman et al. (1995) afirmaram que, em função do processamento, a extrusão da soja integral pode facilitar a atuação de enzimas, favorecendo sua hidrólise e promovendo aumento na disponibilidade dos nutrientes.

A mortalidade dos frangos na fase final (36 a 45 dias de idade) foi de $3,52 \%$. Da mesma forma que na fase de crescimento, não houve efeito $(\mathrm{P}>0,05)$ dos tratamentos sobre o consumo de ração $(\mathrm{CR})$, o ganho de peso (GP) e a conversão alimentar (CA), conforme demonstrado na Tabela 3. Resultados semelhantes foram obtidos por Pinheiro (1993), que testou a substituição do farelo de soja e óleo por soja integral extrusada (SIE) e tostada (SIT) e não encon- 
Tabela 2 - Efeitos dos níveis de temperatura de extrusão da soja integral na ração sobre o consumo de ração (CR), o ganho de peso (GP) e a conversão alimentar (CA) de frangos de corte machos da linhagem Ross durante a fase de crescimento (22 a 35 dias de idade)

Table 2 - Effects of dietary full-fat extrude soybean at different temperature levels on feed intake (FI), weight gain (WG) and feed:gain ratio (FGR) of Ross male broilers during the growing phase (22 to 35 days old)

\begin{tabular}{lccc}
\hline Temperatura $\left({ }^{\circ} \mathrm{C}\right)$ & $\mathrm{CR}(\mathrm{g})$ & $\mathrm{GP}(\mathrm{g})$ & $\mathrm{CA}(\mathrm{g} / \mathrm{g})$ \\
Temperature & $F I$ & $W G$ & $F G R$ \\
\hline 125 & 2000 & 1103 & 1,813 \\
130 & 2012 & 1091 & 1,844 \\
135 & 1993 & 1069 & 1,864 \\
140 & 1995 & 1085 & 1,839 \\
Média (Average) & 2000 & 1087 & 1,840 \\
Regressão (Regression) & $\mathrm{NS}$ & $\mathrm{NS}$ & $\mathrm{NS}$ \\
CV\% & 1,44 & 7,78 & 6,13 \\
\hline
\end{tabular}

NS = Não-significativo $(P>0,05)$ pelo teste $F$. $N S=$ Not significant $(P>0.05)$ effect by $F$ test.

Tabela 3 - Efeitos dos níveis de temperatura de extrusão da soja integral na ração sobre o consumo de ração (CR), o ganho de peso (GP) e a conversão alimentar (CA) de frangos de corte machos da linhagem Ross na fase final (36 a 45 dias de idade)

Table 3 - Effects of dietary full-fat extrude soybean at different temperature levels on feed intake (FI), weight gain (WG) and feed:gain ratio (FGR) of Ross male broilers during the growing phase ( 36 to 45 days old)

\begin{tabular}{lccc}
\hline Temperatura $\left({ }^{\circ} \mathrm{C}\right)$ & $\mathrm{CR}(\mathrm{g})$ & $\mathrm{GP}(\mathrm{g})$ & $\mathrm{CA}(\mathrm{g} / \mathrm{g})$ \\
Temperature & $F I$ & $W G$ & $F G R$ \\
\hline 125 & 1540 & 591 & 2,606 \\
130 & 1499 & 570 & 2,630 \\
135 & 1536 & 551 & 2,788 \\
140 & 1564 & 572 & 2,734 \\
Média (Average) & 1535 & 571 & 2,689 \\
Regressão (Regression) & $\mathrm{NS}$ & $\mathrm{NS}$ & $\mathrm{NS}$ \\
CV\% & 5,34 & 12,53 & 9,34
\end{tabular}

NS = Não-significativo $(P>0,05)$ pelo teste $F$. $N S=$ Not significant $(P>0.05)$ effect by $F$ test.

trou diferença no ganho de peso e no consumo de ração.

Faria (1994) não verificou diferença na conversão alimentar entre grupos tratados com sojas tostada e extrusada. Perilla et al. (1997), no entanto, estudaram o efeito da temperatura de processamento de extrusão úmida $\left(118,120,122,126\right.$ ou $140^{\circ} \mathrm{C}$ em um tempo de 20') sobre a qualidade da proteína e a eficiência alimentar nos frangos e constataram que a tempera- tura de $126^{\circ} \mathrm{C}$ reduziu o conteúdo de fatores antitripsínicos em $97 \%$ (1,36 vs $37,91 \mathrm{mg} / \mathrm{g}$ para soja crua) e melhoraram o crescimento e a conversão alimentar em 27 e $31 \%$, respectivamente. A temperatura de $140^{\circ} \mathrm{C}$ destruiu por completo estes fatores antinutricionais, mas reduziu a solubilidade da proteína em $\mathrm{KOH}$ até $67 \%$, nível considerado inaceitável.

A susceptibilidade das aves aos inibidores presentes no grão cru diminui com a idade e provavelmente com o tempo de exposição (Saxena et al., 1963). Alumot \& Nitsan (1961) determinaram que a atividade proteolítica no intestino dos frangos que receberam soja crua permaneceu praticamente inibida até a $3^{\mathrm{a}}$ semana de vida. A partir da $4^{\mathrm{a}}$ semana, a atividade se recuperou até alcançar níveis quase normais; porém, a hipertrofia pancreática praticamente continuou, podendo se observada ainda na $8^{\mathrm{a}}$ semana.

É provável que a falta de efeitos significativos dos níveis de temperatura de extrusão sobre o desempenho observada neste trabalho esteja relacionada à idade das aves, à maturidade do sistema enzimático e ao tempo de processamento da extrusão.

Lee \& Garlich (1992) compararam o processo térmico padrão do farelo de soja em condições mais severas, como forma de aumentar a temperatura e o tempo de processamento de 50 a $75 \%$ do valor considerado normal e observaram que o superprocessamento reduzia a disponibilidade dos aminoácidos em frangos em crescimento, enquanto, em galos, esse efeito não foi constatado, indicando que as aves adultas suportam melhor que as jovens à soja tratada a temperaturas e tempos de retenção superiores aos níveis ótimos comerciais.

Lee etal.(1991), em trabalho semelhante, concluíram que a temperatura de processamento e o tempo de retenção do farelo de soja podem elevar de 88 a $104^{\circ} \mathrm{C}$ em 37 a $50 \mathrm{~min}$, sem afetar o crescimento, existindo diferenças quanto à sensibilidade dos animais ao superprocessamento da soja. Um ligeiro excesso de calor reduz ao mínimo os fatores antitrípsicos presentes no grão, o que poderia justificar a menor disponibilidade dos aminoácidos (Zarkadas \& Wiseman, 2000).

As médias dos resultados de rendimentos de carcaça eviscerada ( $\mathrm{RC}$ - sem pena e vísceras comestíveis e não-comestíveis), de peito (RP) e de perna $(\mathrm{RPN}=$ coxa e sobrecoxa) e da gordura abdominal (GA) (Tabela 4) não foram afetadas $(\mathrm{P}>0,05)$ pelos tratamentos experimentais da soja integral extrusada. 
Tabela 4 - Efeitos dos níveis de temperatura de extrusão da soja integral na ração sobre os rendimentos de carcaça $(R C)$, de peito (RP) e de perna (RPN = coxa e sobrecoxa) e a gordura abdominal (GA) de frangos de corte machos da Linhagem Ross durante a fase final (36 a 45 dias de idade)

Table 4 - Effects of dietary full-fat extrude soybean at different temperature levels on yields of carcass $(C Y)$, breast yield (BY) and leg - things and drumsticks ( $L Y)$ and abdominal fat (AF) of Ross, males broilers during the finishing phase ( 36 to 45 days old)

\begin{tabular}{lcccc}
\hline Temperatura $\left({ }^{\circ} \mathrm{C}\right)$ & $\mathrm{RC}(\%)$ & $\mathrm{RP}(\%)$ & $\mathrm{RPN}(\%)$ & $\mathrm{GA}(\%)$ \\
Temperature & $C Y$ & $B Y$ & $L Y$ & $A F$ \\
\hline 125 & 78,34 & 29,15 & 28,71 & 2,11 \\
130 & 77,77 & 29,16 & 28,5 & 2,15 \\
135 & 78,68 & 28,96 & 28,94 & 2,12 \\
145 & 78,03 & 29,65 & 28,15 & 2,05 \\
Média & 78,21 & 29,23 & 28,56 & 2,11 \\
Average & & & & \\
Regressão & $\mathrm{NS}$ & $\mathrm{NS}$ & $\mathrm{NS}$ & $\mathrm{NS}$ \\
Regression & & & & \\
CV\% & 1,38 & 2,25 & 2,46 & 12,92 \\
\hline
\end{tabular}

NS $=$ Não-significativo $(P>0,05)$ pelo teste $F$. $N S=$ Not significant $(P>0.05)$ effect by $F$ test.

Não foi observado efeito dos tratamentos sobre as características de carcaça $(\mathrm{P}>0,05)$, o que está de acordo com os resultados obtdios por Sakomura et al. (1998) e Leeson et al. (1987), ao compararem as sojas integral extrusada e tostada com o farelo de soja + óleo.

Os parâmetros de carcaça estudados não diferiram estatisticamente entre os tratamentos, sugerindo que futuros estudos utilizando maiores amplitudes térmicas de processamento da soja integral sejam realizados para se esclarecer melhor os efeitos da inclusão da soja integral extrusada sobre o desempenho de frangos de corte, considerando-se também os resultados do teste de urease e da solubilidade da proteína em $\mathrm{KOH}$.

\section{Conclusões}

A soja integral extrusada, após processamento à temperatura entre 125 e $140^{\circ} \mathrm{C}$, pode ser utilizada em rações para frangos de corte nas fases de crescimento e final, sem causar prejuízos no desempenho das aves.

\section{Agradecimento}

À Empresa GUARAVES (Guarabira Aves Ltda).

\section{Literatura Citada}

ALUMOT, E.; NITSAN, Z. The influence of soybean antitrypsin on the intestinal proteolysis of the chick. Journal of Nutrition, v.73, p.71-77, 1961.

BOND, D.A.; SMITH, D.B. Possibilities for the reduction of antinutritional factors in grain legumes by breeding. In: HUISMAN, J.; van der POEL; A.F.B.; LIENER, I.E. (Eds.) Recent advances of research in antinutritional factors in legume seeds. Wageningh: Pudoc, 1988. p.285-296.

BUTOLO, J.E. Fatores ligados à alimentação e nutrição de reprodutores. In: CONFERÊNCIA APINCO 2001 DE CIÊNCIA E TECNOLOGIA AVICOLAS, 2001, Campinas. Anais... Campinas: Fundação Apinco de Ciência e Tecnologia Avícolas, 2001. p.125-146.

FARIA, D.E. Avaliação nutricional de soja integral tostada e extrusada para poedeiras comerciais. Jabuticabal, 1994. 103p. Dissertação (Mestrado em Zootecnia) - Universidade Estadual Paulista, 1994.

HAN, Y.; PARSONS, C.M.; HYMOWITZ, T. Nutritional evaluation of soybeans varying in trypsin inhibitor content. Poultry Science, v.70, p.896-906, 1991.

JEROCH, H.; DÄNICKE, S. Barley in poultry feeding: a review. World's Poultry Science Journal, v.51, p.271-291, 1995.

LEE, H.; GARLICH, J.D.; FERKET, P.R. Effect of over coocked soybean meal on turkey performance. Poutry Science, v.70, p.2509-2515, 1991.

LEE, H.; GARLICH, J.D. Effect of over coocked soybean meal on chicken performance and amino acid availability. Poultry Science, v.71, p.499-508, 1992.

LEESON, S.; ATTEH, J.O.; SUMMERS, J.D. Effects increasing dietary levels of commercial heated soybeans on performance, nutrient retention and carcass quality of broiler chickens. Canadian Journal Animal Science, v.67, p.821-825, 1987.

LIENER, I.E. The lectins: properties, functions and applications in biology and medicine. Orlando: Academic Press, 1986.

LUDKE, M.C.M.M.; DE LIMA, G.L.M.M.; LANZNASTER, M. et al. Efeito do tipo de processamento da soja integral sobre o desempenho, qualidade de carcaça e valorização econômica de suínos dos $25 \mathrm{~kg}$ ao abate. In: REUNIÃO ANUAL DA SOCIEDADE BRASILEIRA DE ZOOTECNIA, 41., 2004, Campo Grande. Anais... Campo Grande-MS: Sociedade Brasileira de Zootecnia, 2004. 6p. CD-ROM. Nutrição de Não-Ruminantes.

MARSMAN, G.J.P.; GRUPPEN, H.; VANDER POEL, A.F.B. et al. The effect of shears forces and addition of a mixture of a protease and physiological parameters during extrusion of siybean meal. Animal Feed Science Technology, v.56, n.1 2, p.21-35, 1995 .

MOREIRA, I. Utilização de soja grão processada e suas implicações na alimentação de monogástricos. In: CONGRESSO NORDESTINO DE PRODUÇÃO ANIMAL, 1., SIMPÓSIO NORDESTINO DE ALIMENTAÇÃO DE RUMINANTES, 2., Teresina. Anais... Teresina: 2000. v.7, p.223-229.

PERILLA, N.S.; CRUZ, M.P.; De BELALCAZAR, F. et al. Effect of temperature of wet extrusion on the nutritional value of full-fat soybeans for broiler chickens. British Poultry Science, v.38, p.412-416, 1997.

PINHEIRO, J.W. Soja integral processada pelo calor em rações de frangos de corte. Jaboticabal: Universidade Estadual 
Paulista, 1993, 175p. Tese (Doutorado em Zootecnia) Universidade Estadual Paulista, 1993.

ROSTAGNO, H.S.; DIONIZIO, M.A.; ALBINO, L.F.T. Perspectivas da nutrição de frangos de corte. In: REUNIÃO ANUAL DA SOCIEDADE BRASILEIRA DE ZOOTECNIA, 41., 2004, Campo Grande. Anais... Campo Grande: Sociedade Brasileira de Zootecnia, 2004. 17p. CDROM. Nutrição de Não-Ruminantes.

ROSTAGNO, H.S.; SILVA, D.J.; COSTA, P.M.A. et al. Composição de alimentos e exigências nutricionais de aves e suínos (Tabelas Brasileiras). Viçosa, MG: Universidade Federal de Viçosa, 2000. 141p.

SAKOMURA, N.K.; SILVA, R.; LUCEUSENTZ, A.C. et al. Avaliação da soja integral tostada ou extrusada sobre o desempenho de frangos de corte. Revista Brasileira de Zootecnia, v.27, n.3, p.584-594, 1998.

SAID, N. Introduction to dry extrusion, full fat soybean processing. In: PRACTICAL SHORT COURSE MANUAL ON FEEDS AND PET FOOD EXTRUSION, p.1-44. Texas A\&M University, College Station, 1999.
SAXENA, H.C.; JENSEN, L.S.; Mc GINNIS, J. Pancreatic hypertrophy na chick grow inhibition by soybean factores devoid of trypsin inhibitor. Proceedings of the Society of Experimental Biology and Medicine, v.112, p.101-105, 1963.

UNIVERSIDADE FEDERAL DE VIÇOSA/UFV. SAEG Sistema de Análises Estatísticas. Versão 8.0 Viçosa, MG: 1999. 69p.

ZARKADAS, L.N.; WISEMAN, J. Inclusion of full fat soybean in piglet diets. Proceedings of the British Society Animal. Science Ocasional Meeting: the weaner pig, 2000. p.45-46.

Recebido em: 19/07/04

Aceito em: 16/06/05 